\title{
A STOP LOSS INEQUALITY FOR COMPOUND POISSON PROCESSES WITH A UNIMODAL CLAIMSIZE \\ DISTRIBUTION
}

\author{
H. G. VERBEEK \\ Amsterdam
}

\section{INTRODUCTION}

The paper considers the problem of finding an upper bound for the Stop loss premium.

We will start with a brief sketch of the practical context in which this problem is relevant.

If it is reasonable to assume, that the accumulated claims variable of the underlying risk can be represented by a Compound Poisson Process, the following data are needed for fixing the Stop loss premium:

- the claims intensity,

- the distribution of the claimsizes (jump-size variable).

In practical situations it is usually possible to find a reasonable estimate for the claims intensity (expected number of claims in a given period).

Generally speaking, however, it is not so easy to get sufficient data on the claimsize distribution. Ordinarily only its mean is known. This deficiency in information can of course be offset by assuming the unknown distribution to be one of the familiar types, such as Exponential, Gamma or Pareto.

Stop loss premiums are however very sensitive to variations in the type of claimsize distribution and consèquently it can make a lot of difference in the result what particular choice is made.

To gain some insight into the consequences of a specific assumption, it is useful to know within what range the premium can move for varying distributional suppositions. This means establishing an upper bound and a lower bound. The lower bound is trivially obtained if the mass of the claimsize distribution is solely concentrated at its mean. The upper bound on the other hand should 
correspond to the "worst" possible claimsize distribution. This means, that we have to look for a distribution which maximizes the Stop loss premium.

Thus posed the question could be interpreted as a problem in Variational Calculus.

An actual approach to this problem by Gagliardi/Straub [I] and Bühlmann [2] has been along different lines. They start with an assumption [3] for the maximizing distribution and subsequently prove the truth of their assumption.

It is intuitively clear that a condition for the existence of a maximizing distribution is, that the claimsize variable be restricted to a finite interval. An assumption which is consequently made in the papers mentioned.

We will prove in this paper that by making the additional assumption of unimodality, a reduction of the upper bound as found in the cited papers can be accomplished.

In a paper by Gerber [4] it is rightly argued that unimodality can realistically be imposed on many distributions which are relevant in the insurance field.

\section{Some Definitions}

For easy reference we cite the following:

Definition 2.I: a realvalued function $F$ defined on an interval $I$ of the real line is convex on $I$ if, for any two points $x$ and $y$ in $I$ and any number $t$ such that $0<t<\mathrm{I}$,

$$
F[t x+(I-t) y] \leq t F(x)+(\mathrm{I}-t) F(y)
$$

The function $F$ is concave if the inequality sign is reversed.

From [5] page I55 we quote:

Definition 2.2: a distribution function $F$ is unimodal with the mode at the origin if the graph of $F$ is convex in $(-\infty, 0)$ and concave in $(0, \infty)$.

The unimodality requires that $F$ is continuous with a possible exception at the origin.

Note: in what follows we will assume that the definition of unimodality implies continuity in the entire closed interval in which $F$ is defined. 
Further we quote from [2] the following:

Definition 2.3:

- $Y$ representing a non-negative r.v. with maximum $M$ and $\operatorname{df} G(x)$; hence $G(-0)=0$ and $G(M)=\mathrm{I}$; and

$-Y^{*}$ a modified r.v. taking on only the two values $O$ and $M$ with probabilities $I-p$ and $p$.

In addition it is required that $E(Y)=E(Y)^{*}=p M$. It is shown in [I] and [2] that the Stop loss premium based on $Y^{*}$ as the claimsize variable will always exceed or equal the premium based on $Y$.

3. A Maximizing Randon Variable for Unimodal Claimsize VARIABLES

We introduce the following random variables:

Definition 3.I:

$-Z$ a non-negative r.v. with maximum $M$ and $d f G(x)$ supposed unimodal with the mode at $m(0<m<M)$ and $G(0)=0$; hence $G(-0)=0$ and $G(M)=I$; and

$-Z^{*}$ a modified r.v. with $d f$

$$
\begin{array}{ll}
G^{*}(x)=I-2 p+2 p x \mid M & \text { for all } x \in[0, M] \\
G^{*}(x)=0 & \text { otherwise }
\end{array}
$$

We also require $p<0.5$ and $E(Z)=E\left(Z^{*}\right)=p M$.

We shall show in section 4 that the variable $Z^{*}$ accomplishes an upper bound if replacing $Z$ as a claimsize variable in the Stop loss premium. We will also show, that the upper bound produced by $Z^{*}$ is at most as high as that of $Y^{*}$. To prove this we shall later need the following:

Lemma $I:$ if $a$ is an arbitrary real number and $E(Y)=E(Z)$ then

$$
E\left[\left(Z^{*}-a\right)^{+}\right] \leq E\left[\left(Y^{*}-a\right)^{+}\right] \text {. }
$$

Proof:

If $a$ is not in $[0, M]$ the inequality is obviously true.

If $a$ is in $[0, M]$ we get:

$$
\begin{aligned}
& E\left[\left(Z^{*}-a\right)^{+}\right]=\int_{a}^{\infty} P\left[Z^{*} \geq x\right] d x=p(I-(a / M I))(M-a) \\
& E\left[\left(Y^{*}-a\right)^{+}\right]=\int_{a}^{\infty} P\left[Y^{*} \geq x\right] d x=p(M-a)
\end{aligned}
$$


It follows that

$$
E\left[\left(Z^{*}-a\right)^{+}\right]=(\mathrm{r}-(a / M)) E\left[\left(Y^{*}-a\right)^{+}\right] .
$$

In view of $0 \leq a \leq M$, the lemma is true.

\section{Auxiliary Lemmas and Main Result}

For the proof of the fact that $Z^{*}$ produces an upper bound with regard to $Z$ we need the following lemmas:

Lemma 2: there exists exactly one number $s$ in $[0, M)$ for which holds:

$$
P[Z \geq s]=P\left[Z^{*} \geq s\right], s \in[0, M) .
$$

Note that we have excluded the number $M$ from $[0, M]$ for which (7) is true by definition.

To increase readability we subdivide the proof in 4 assertions: Assertion $I$ : at least one number satisfying (7) exists in $[0, M)$. Proof: suppose that no such number existed. In view of the continuity of $G$ and $G^{*}$ in $[0, M)$ and the fact that $P[Z \geq 0]>P\left[Z^{*} \geq 0\right]$, we must have in that case:

$$
P[Z \geq x]>P\left[Z^{*} \geq x\right], \text { for all } x \in[0, M)
$$

it follows that

$$
\int_{0}^{M} P(Z \geq x] d x>\int_{0}^{M} P\left[Z^{*} \geq x\right] d x
$$

or equivalently:

$$
E(Z)>E\left(Z^{*}\right) \text {. }
$$

This contradicts $E(Z)=E\left(Z^{*}\right)$ as required in accordance with the definition 3.I of $Z^{*}$ in section 3. Therefore the assertion is true.

Assertion 2: not more than one number satisfying (7) exists in $[0, m]$. (By definition $m$ denotes the mode of $G$ ).

Proof: assume there exist two such numbers $s_{1}$ and $s_{2}$ and let $s_{2}$ be the greater of the two.

In the interval $\left[0, s_{2}\right]$ we can write $G\left(t s_{2}\right)$ in the following manner:

$$
G^{*}\left(t s_{2}\right)=(I-t) G^{*}(0)+t G^{*}\left(s_{2}\right), \text { for all } t \in[0, I]
$$


Since by definition $G(0)=0$ and the unimodality of $G$ implies convexity in $(0, m)$ it follows $(\mathrm{I})$ that:

$$
G\left(t s_{2}\right) \leq t G\left(s_{2}\right) \quad t \in[0, I]
$$

Since for $s_{2}$ identity (7) holds, we have:

$$
G\left(s_{2}\right)=G^{*}\left(s_{2}\right) \text {. }
$$

From (IO), (II) and (I2) we derive:

$$
G^{*}\left(t s_{2}\right) \geq G\left(t s_{2}\right) \quad t \in[0, I] .
$$

Equality holding only for $t=\mathrm{I}$, it is clearly impossible that a number $s_{1}\left(<s_{2}\right)$ exists in $[0, m]$ for which $G^{*}\left(s_{1}\right)=G\left(s_{1}\right)$. This contradicts our initial assumption and proves the assertion.

Assertion 3: not more than one number satisfying (7) exists in $[m, M)$. Except for minor changes the proof is analogous to that of assertion 2.

Assertion 4: there cannot exist two numbers one belonging to $[0, m]$ and one belonging to $(m, M)$, which both satisfy $(7)$.

Proof: assume to the contrary that two such numbers $s_{1}$ and $s_{2}$ exist. We then can write:

$$
\begin{array}{ll}
s_{1}=t_{1} m, & t_{1} \in[0, \mathrm{I}] \\
s_{2}=\left(\mathrm{I}-t_{2}\right) m+t_{2} M, & t_{2} \in(\mathrm{O}, \mathrm{I}) .
\end{array}
$$

Again recalling the definition 3.I of $G^{*}$, we note that:

$$
G^{*}\left(s_{1}\right)=G^{*}\left(t_{1} m\right)=\left(I-t_{1}\right) G^{*}(0)+t_{1} G^{*}(m)
$$

and

$$
G^{*}\left(s_{2}\right)=G^{*}\left[\left(\mathrm{I}-t_{2}\right) m+t_{2} M\right]=\left(\mathrm{I}-t_{2}\right) G^{*}(m)+t_{2} G^{*}(M)
$$

by assumption:

$$
\begin{aligned}
& G\left(s_{1}\right)=G^{*}\left(s_{1}\right) \\
& G\left(s_{2}\right)=G^{*}\left(s_{2}\right)
\end{aligned}
$$

using (I8) we can write for (I6):

$$
G\left(s_{1}\right)=\left(\mathrm{I}-t_{1}\right) G^{*}(0)+t_{1} G^{*}(m)
$$

in the same manner, combining (I 7 ) and (Ig):

$$
G\left(s_{2}\right)=\left(I-t_{2}\right) G^{*}(m)+t_{2} G^{*}(M) \text {. }
$$


On the other hand, because of the assumed unimodality we have the two inequalities:

$$
\begin{aligned}
G\left(s_{1}\right) & =G\left(t_{1} m\right) \leq t_{1} G(m) \quad t_{1} \in[0, m] \\
G\left(s_{2}\right) & =G\left[\left(\mathrm{I}-t_{2}\right) m+t_{2} L\right]>\left(I-t_{2}\right) G(m)+t_{2} G(L)
\end{aligned}
$$

Comparing (20) and (22) we find:

$$
\left(\mathrm{I}-t_{1}\right) G^{*}(0)+t_{1} G^{*}(m) \leq t_{1} G(m) \quad t_{1} \in[0, \mathbf{I}] .
$$

As by definition (2) $G^{*}(0)>0$, we conclude:

$$
G^{*}(m)<G(m) \text {. }
$$

Comparing now (2I) and (23) and noting that $G(M)=G^{*}(M)=I$ it is seen that:

$$
\left(\mathrm{I}-t_{2}\right) G^{*}(m)+t_{2}>\left(\mathrm{I}-t_{2}\right) G(m)+t_{2} t_{2} \in(\mathrm{O}, \mathrm{I})
$$

from (26) we derive finally:

$$
G^{*}(m)>G(m) \text {. }
$$

As the inequalities (25) and (27) contradict each other our initial assumption is proved untrue, which proves the assertion.

The 4 assertions which have been shown to be true prove the lemma 2.

Lemma 3 : if $s$ is the number satisfying (7) then the following inequalities hold:

$$
\begin{aligned}
& P[Z \geq x] \geq P\left[Z^{*} \geq x\right], \text { for all } x \in[0, s] \\
& P[Z \geq x] \leq P\left[Z^{*} \geq x\right], \text { for all } x \in[s, M L) .
\end{aligned}
$$

Proof: follows from lemma 2, the continuity of $G$ and $G^{*}$ and the fact that $P[Z \geq 0]>P\left[Z^{*} \geq 0\right]$.

Lemma 4: for $Z$ and $Z^{*}$ as defined and arbitrary $a$ the following inequality holds:

$$
E\left[(Z-a)^{+}\right] \leq E\left[\left(Z^{*}-a\right)^{+}\right] .
$$

Proof: for $a \leq 0$ and $a \geq M$ the inequality is trivially true.

If $a \in[0, s]$ we write:

$$
\begin{aligned}
& E\left[(Z-a)^{+}\right]=\int_{a}^{\infty} P[Z \geq x] d x \\
= & \int_{0}^{u} P[Z \geq x] d x-\int_{0}^{a} P[Z \geq x] d x
\end{aligned}
$$




$$
\begin{aligned}
& \text { using } E(Z)=E\left(Z^{*}\right) \text { and }(28) \\
& \qquad \begin{array}{l}
\leq \int_{0}^{M} P\left[Z^{*} \geq x\right] d x-\int_{0}^{a} P\left[Z^{*} \geq x\right] d x \\
\quad=\int_{a}^{M} P\left[Z^{*} \geq x\right] d x=E\left[\left(Z^{*}-a\right)^{+}\right]
\end{array}
\end{aligned}
$$

If $a \in(s, M)$ we make use of (29) and note:

$$
E\left[(Z-a)^{+}\right]=\int_{a}^{x} P[Z \geq x] d x \leq \int_{a}^{M} P\left[Z^{*} \geq x\right] d x
$$

which is equivalent to (30).

This proves. the lemma.

In [2] it is shown that:

$$
E\left[\left(S_{n}-A\right)^{+}\right] \leq E\left[\left(S_{n}^{*}-A\right)^{+}\right]
$$

where $S_{n}=\sum_{i=1}^{n} Y_{i}$ and $S_{n}^{*}=\sum_{i=1}^{n} Y_{i}^{*}$

if $Y_{1}, Y_{2}, \ldots, Y_{n}, Y_{1}^{*}, Y_{2}^{*}, \ldots, Y_{n}^{*}$ are independently distributed variables conforming to definition 2.3.

In [2] this result is obtained as an immediate consequence of the inequality (30) with $Z$ and $Z^{*}$ replaced by $Y$ and $Y^{*}$. Since for $Z$ and $Z^{*}$, according to Lemma 4 , the same inequality holds, the result is also true for $Z$ and $Z^{*}$.

Thus we have:

Lemma 5: for $Z_{1}, Z_{2}, \ldots, Z_{n}, Z_{1}^{*}, \ldots, Z_{n}^{*}$ independent, each $Z_{i}$ distributed with unimodal d.f. and each $Z_{i}^{*}$ according to (2), all in accordance with the definitions of $Z$ and $Z^{*}$, given in section 3 and $A$ an arbitrary number, we have:

$$
E\left[\left(S_{n}-A\right)^{+}\right] \leq E\left[\left(S_{n}^{*}-A\right)^{+}\right]
$$

with

$$
S_{n}=\sum_{i=1}^{n} Z_{i}, S_{n}^{*}=\sum_{i=1}^{n} Z_{i}^{*}
$$

Theorem: let $W_{t}$ be a Compound Poisson process with claimsize distribution $G(x)$ and $W_{t}^{*}$ a Compound Poisson process with distribution $G^{*}(x)$. 
If $G$ and $G^{*}$ are as defined in section 3 and $W_{t}$ and $W_{t}^{*}$ have the same claims intensity $\lambda$, then:

$$
E\left[\left(W_{t}-A\right)^{+}\right] \leq E\left[\left(W_{t}^{*}-A\right)^{+}\right] .
$$

Proof: as observed in [2] the proof follows because (32) holds for each fixed number of claims in consequence of (3I).

The theorem proves that replacing an unimodal claimsize variable $Z$ by a modified variable $Z^{*}$, both according to definition $3 . I$, results in an upper bound for the Stop loss premium, if the counting variable can be represented by a Poisson process. From the proof it is clear, that the validity of the theorem is actually not restricted to Poisson counting variables, but that it holds for all discrete non-negative distributions.

Proposition: the upper bound according to $Z^{*}$ as stated in the RHS of (32) is smaller or at the most equal to the upper bound resulting from $Y^{*}$.

Proof: follows by applying to Lemma I the argument leading to Lemma 5 and subsequent use of the theorem.

\section{Numerical Evaluation of the Upper Bound}

We will now derive an expression which permits the numerical evaluation of the upper bound as stated in the RHS of (32). To simplify the algebra we will make use of the Laplace transform technique.

If $g^{*}(x)$ denotes the density of:

$$
\begin{aligned}
& G^{*}(x)=I-2 p+2 p x \mid M \text { for all } x \in[0, M] \\
& G^{*}(x)=0 \text { otherwise }
\end{aligned}
$$

we define:

$$
L\left[g^{*} ; s\right]=\int_{0}^{\infty} e^{-x s} d G^{*}(x)=\mathrm{I}-2 p+2 p\left(\mathrm{I}-e^{-M s}\right) \mid M s .
$$

Employing $F(x)$ for the distribution of $W_{t}^{*}$ we find:

$$
L[(I-F) ; s]=s^{-1}-\exp \left\{-\lambda t+\lambda t L\left[g^{*} ; s\right]\right\} \mid s .
$$

Substituting (34) in (35) and writing $c=2 p \lambda t$ for short, we get:

$$
L[(I-F) ; s]=s^{-1}-\exp \left[-c+c\left(\mathrm{I}-e^{-M s}\right) \mid M s\right] \mid s . \quad(36)
$$


We now introduce the abbreviation:

$$
E^{*}=E\left[\left(W_{t}^{*}-A\right)^{+}\right]
$$

and take the Laplace transform of $E^{*}$ with respect to $A$. This gives:

$$
L\left[E^{*} ; s\right]=\{0.5 c M-L[(\mathrm{I}-F) ; s]\} \mid s
$$

after substitution of $\left(3^{6}\right)$ in (38) we obtain:

$L\left[E^{*} ; s\right]=\left\{-\mathrm{I}+0.5 c M s+\exp \left[-c+c\left(\mathrm{I}-e^{-M s}\right) \mid M s\right]\right\} \mid s^{2}$.

To invert (39) we develop the RHS in powers of $\exp (-M s)$ and find:

$$
\begin{gathered}
L\left[E^{*} ; s\right]=-s^{-2}+0.5 c M s^{-1}+ \\
e^{-c}\left[s^{-2} e^{(c / s M)}-\frac{c s^{-3}}{M} e^{(c i s M)-M s}+\frac{c^{2} s^{-4}}{2 ! M^{2}} e^{(c / s M)-2 M s}-\ldots\right]
\end{gathered}
$$

The RHS can be inverted into hyperbolic Bessel functions of ascending order, by using the following standard result:

$L\left[\left\{\frac{(x-j M)^{+}}{c}\right\}^{(n-1) / 2} I_{n-1}\left\{2 \sqrt{c(x-j M)^{+}}\right\} ; s\right]=s^{-n} e^{(c / s)-j M s}$

Applying (4I) to the RHS of (40) term by term and writing $k=A \mid M$ for short gives:

$$
E^{*} \mid M=-k+0.5 c+\frac{e^{-c}}{c} \sum_{n=0}^{\infty} \frac{(-I)^{n}}{n !}\left[c(k-n)^{+}\right]^{(n+1) / 2} I_{n+1}\left[2 \sqrt{c(k-n)^{+}}\right]
$$

In (42) we have introduced $k$ which is the deductible (excess point) of the Stop loss reinsurance expressed in the maximum of the single risks. If $k$ is a positive integer we can simplify (42) as follows:

$E^{*} \mid M=-k+0.5 c+\frac{e^{-c}}{c} \sum_{n=0}^{k} \frac{(-I)^{n}}{n !}\left[c(k-n)^{+}\right]^{(n+1 / 2)} I_{n+1}\left[2 \sqrt{c(k-n)^{+}}\right]$

The finite series (43) represents the bound of the Stop loss premium expressed in the maximum $M$. 


\section{CONCLUDING REMARK}

With the help of standard tables for Bessel functions, for example in [6] actual calculation of the bound is quite easy in practice.

\section{REFERENCES}

[I] Gagliardi und Straub (I974): Eine obere Grenze für Stop-LossPrämien, MVSV 1974, 21 5-221.

[2] BühlmaNo (1974): Ein anderer Beweis für die Stop-Loss-Ungleichung in der Arbeit Gagliardi/Straub, MVSM 1974, 284-285.

[3] Benktander (1974): A Motor Excess Rating Problem: Flat Rate with Refund, (ASTIN Colloquium, Turku, Finland).

[4] Gerber (r972): Ein Satz von Khintchin und die Varianz von unimodalen Verteilungen, MVSV 1972, 225-331.

[5] FELLER: An Introduction to Probability Theory and its Applications, Vol. 2, Wiley I966.

[6] Watson: Theory of Bessel functions, Cambridge U.P. I966 (reprint) 